\title{
Object-based attention determines dominance in binocular rivalry
}

\author{
Jude F. Mitchell ${ }^{*}$, Gene R. Stoner ${ }^{\dagger}$, \& John H. Reynolds ${ }^{*}$ \\ * Systems Neurobiology Laboratory, + Vision Center Laboratory, The Salk Institute for Biological Studies, La Jolla, CA, 92037 USA.
}

A question of longstanding interest to philosophers, psychologists, and neuroscientists is how the brain selects which signals enter consciousness. Binocular rivalry and attention both involve selection of visual stimuli, but impact perception quite differently. During rivalry, awareness alternates between two different stimuli presented to the two eyes. In contrast, attending to one of two stimuli impairs discriminations of the ignored stimulus but without causing it to disappear. Here we show that despite this difference, attention and rivalry rely on shared objectbased selection mechanisms. We cued attention to one of two superimposed transparent surfaces and then deleted the image of one surface from each eye, resulting in rivalry (Figure 1). Observers usually reported seeing only the cued surface during subsequent rivalrous viewing (Figure 2A). In a second experiment we asked observers to report a second translation that followed during rivalrous viewing and was either of the cued or uncued surface. They were less accurate in judging translations of the uncued surface (Figure 2B). Our design ensured that selection of the cued surface could not have

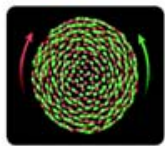

A. Rotation $(750 \mathrm{~ms})$

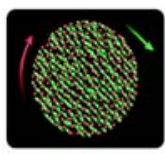

B. Translation (150ms)

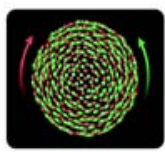

C. Rotation (150ms)

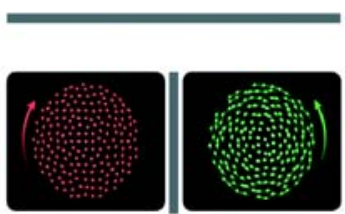

\section{Dichoptic Viewing} (variable period)

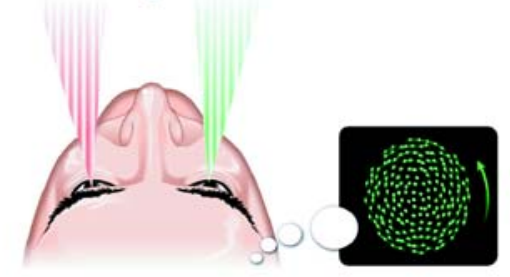

E. Dominance Judgment

Figure 1 Dominance-Judgment Task.

Panels are arranged from top to bottom according to the sequence of events in each trial. (A) Two sets of dots rotated in opposite directions yielding a percept of superimposed transparent surfaces. For illustration purposes, we show the surfaces with different colors (red and green), but they were actually the same color. (B) One surface translated for $150 \mathrm{~ms}$ in one of eight directions, while the other surface continued to rotate. Subjects reported the direction of translation. (C) Both surfaces resumed rotation for $150 \mathrm{~ms}$. (D) The image of one surface was removed from each eye, resulting in rivalry. Subjects judged which surface was dominant after a variablelength period of rivalrous viewing. (E) Observers usually perceived the previously translated surface as dominant. resulted from spatial, ocular, or feature-based mechanisms. Rather, attention was drawn to one surface, and this caused the other surface to be suppressed during rivalry. These results raise the question of how object representations compete during these two forms of perceptual selection, even as the motion features of those objects change unpredictably over time.
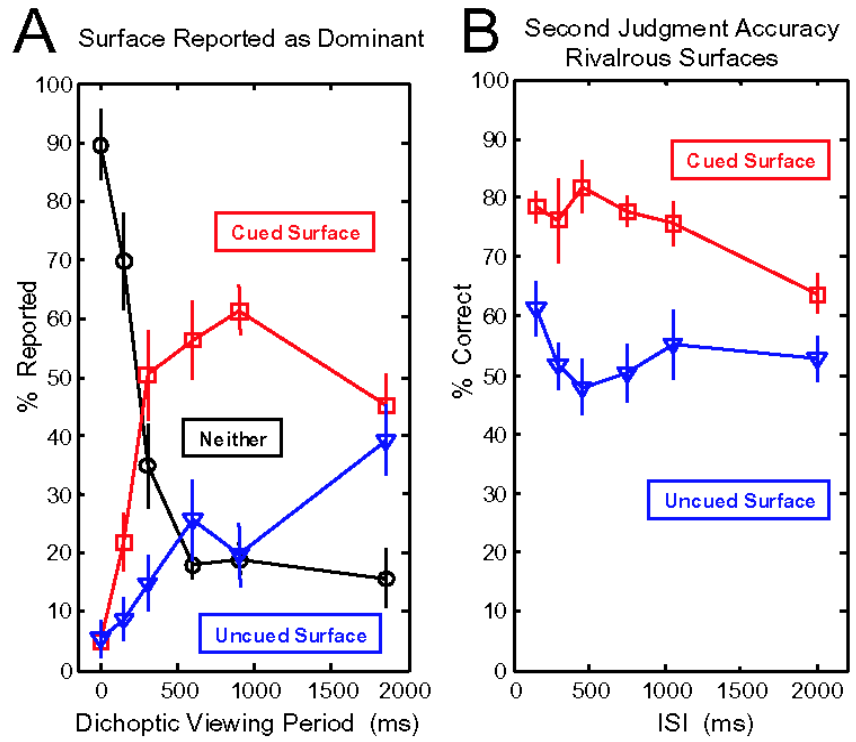

Figure 2 Data from Dominance-Judgment \& Double-Translation Tasks.

(A) Seven observers reported whether either surface was dominant at the end of dichoptic presentation, and if so, which surface was dominant. The mean percentage of trials on which the cued or uncued surface was reported to be dominant is shown in red and blue, respectively. The percentage of trials on which neither surface was clearly dominant appears in black. (B) Mean accuracy in reporting the direction of the second translation averaged across trials in which the surfaces were presented dichoptically. Line color indicates whether the cued (red line) or uncued (blue line) surface translated second. The inter-stimulus interval (ISI) is the delay between the end of the cueing translation and start of the second translation (ISI = dichoptic viewing period $+150 \mathrm{~ms}$ ). Error bars indicate standard errors of the mean (SEM) across subjects. 